\title{
AVALIAÇÃO DO PROCESSO DE TRABALHO ENTRE EQUIPES DE SAÚDE DA FAMÍLIA DE UM MUNICÍPIO DE MINAS GERAIS, BRASIL
}

\author{
EVALUATION OF THE WORK PROCESS OF FAMILY HEALTH TEAMS \\ IN A MUNICIPALITY IN MINAS GERAIS, BRAZIL
}

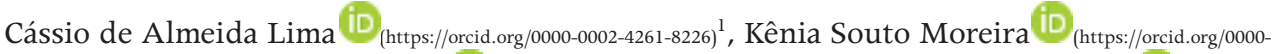 0002-0661-616X)², Gustavo Silva Costa (iD (htps://orcid.org/0000-0003-0844-4610) ${ }^{3}$, Rayane Soares Maia (iD) (https:// orcid.org/0000-0001-7855-6058) 3), Mânia de Quadros Coelho Pinto (Didtps://orcid.org/0000-0002-6913-8695) ${ }^{3}$, Maria Aparecida Vieira (DiDtps://orcid.org/0000-0002-7921-4049) ${ }^{4}$, Simone de Melo Costa (iD) (http://orcid.org/0000-0002-0266- $018 x)^{3}$

\footnotetext{
${ }^{1}$ Universidade Federal dos Vales do Jequitinhonha e Mucuri, Faculdade de Ciências Biológicas e da Saúde, Programa de Pós-Graduação em Saúde, Sociedade e Ambiente, Diamantina, Minas Gerais, Brasil.

$<$ cassioenf2014@gmail.com>

${ }^{2}$ Faculdades Integradas Pitágoras de Montes Claros, Departamento de Medicina, Montes Claros, Minas Gerais, Brasil. ${ }^{3}$ Universidade Estadual de Montes Claros, Centro de Ciências Biológicas e da Saúde, Departamento de Odontologia, Montes Claros, Minas Gerais, Brasil.

${ }^{4}$ Universidade Estadual de Montes Claros, Centro de Ciências Biológicas e da Saúde, Departamento de Enfermagem, Montes Claros, Minas Gerais, Brasil.
}

Resumo Este estudo teve como objetivo avaliar a organização do processo de trabalho entre equipes de saúde da família de um município de Minas Gerais (MG), Brasil. Pesquisa quantitativa, transversal, analítica, desenvolvida em 2014. Investigaram-se formulários da Autoavaliação para Melhoria do Acesso e da Qualidade da Atenção Básica de 73 equipes. Foram considerados 14 itens da subdimensão organização do processo de trabalho, avaliados com valores entre zero e 10 pontos, referindo-se à total inadequação ou adequação da equipe ao padrão avaliado. As equipes foram classificadas do padrão 'muito insatisfatório' ao 'muito satisfatório'. Efetuaram-se análises estatísticas com nível de significância $<<0,05$. Classificaram-se 86,3\% nos padrões muito satisfatório/satisfatório, mas 13,7\% foram regular/muito insatisfatório. Trêsitens - 'Território definido, vínculo com a população e responsabilização pela resolução das necessidades de saúde'; ' 'Coordenação do cuidado' e 'Monitoramento das solicitações de exames' - apresentaram desempenho inadequado. As equipes da zona urbana foram melhor avaliadas. $\mathrm{O}$ maior número de profissionais da odontologia foi correlacionado à maior pontuação. Atenção especial deve ser dada àquelas equipes com baixa pontuação. Melhor organização do processo de trabalho deve ser fortalecida, visando aprimorar a performance no cuidado à saúde da comunidade.

Palavras-chave gestão em saúde; serviços de saúde; avaliação de serviços de saúde; estratégia saúde da família; atenção primária à saúde.
Abstract The study had the goal of assessing the organization of the work process of family health teams in a municipality in the state of Minas Gerais, Brazil. It was a quantitative, cross-sectional analytical research performed in 2014. We investigated the forms for the Self-Assessment for the Improvement in the Access and Quality of Primary Health Care of 73 teams. A total of 14 items of the subdimension organization of the work process were considered, and they were scored from 0 to 10 , with 0 meaning complete inadequacy and 10 meaning complete adequacy of the team to the assessed pattern. The teams were classified from the 'highly unsatisfactory' pattern to the 'highly satisfactory' pattern. The significance level of the statistical analyses was set as values of $\mathrm{p}<0.05$. A total of $86.3 \%$ were classified in the highly satisfactory/satisfactory pattern, and $13.7 \%$ were classified as average/highly unsatisfactory. Three items - 'Defined territory, bond with the population and responsibility for the fulfilment of the health needs'; 'Coordination of care'; and 'Monitoring of exam requests' - had inadequate performances. The teams from urban areas scored better in the assessment. The odontology professionals were the category that scored the highest in the assessment. Special attention should be devoted to the teams with low scores. A better organization of the work process should be strengthened, with the goal of enhancing the performance in the care to health of the community.

Keywords health management; health services; assessment of health services; family health strategy; primary health care. 


\section{Introdução}

No âmbito do Sistema Único de Saúde (SUS), a Atenção Básica (AB) configura um eixo basilar para a organização dos serviços de saúde, tendo como propósito constituir uma das principais alternativas para transformações e avanços no modelo de atenção à saúde. Em seguida à sua implantação e ao desenvolvimento de suas estruturas políticas, operacionais e financeiras, cada vez mais a $\mathrm{AB}$ tem se tornado referência no sistema de saúde brasileiro, inclusive nas políticas e no planejamento em saúde, tanto na esfera de governo municipal, quanto estadual ou federal. Nos últimos anos, a Estratégia de Saúde da Família (ESF) tem assumido especial destaque na reorganização e implementação da $\mathrm{AB}$, com impacto nos referenciais que norteiam a estruturação dos sistemas locais de saúde (Gil, 2006; Leite et al., 2016).

No Brasil, a ESF configura um lócus potente para a reversão da tradicional assistência - restrita a procedimentos individuais - em novas práticas compartilhadas entre equipes. Estas aplicam distintas tecnologias no cuidado e consolidam a clínica ampliada direcionada às necessidades e demandas dos usuários (Gil, 2006; Santos et al., 2015). A ESF apresenta baixo custo, foca na promoção da saúde e prevenção de doenças, almeja maior racionalidade na utilização dos demais serviços de saúde, a fim de ofertar maior satisfação do usuário e qualidade de vida à comunidade. Nos últimos anos, transformou-se no modelo hegemônico da $\mathrm{AB}$ e tem sido indicada como a principal estratégia de enfrentamento da crise da saúde no país (Maciel, Caldeira e Diniz, 2014).

Sendo assim, a ESF apresenta um papel essencial, posto que: deve garantir maior oferta e qualidade das ações de saúde pública; vem se conformando como a principal porta de entrada do SUS; e propicia a humanização por meio de novas relações entre o usuário e a equipe multiprofissional. A implantação da ESF também fomentou o advento de um novo panorama na $\mathrm{AB}$, focado na tríade usuário-família-comunidade, com a garantia de efetividade na assistência (Lima et al., 2014; Leite et al., 2016). Todavia, a consolidação da ESF ainda esbarra em diversos desafios e agudiza as dificuldades no interior do SUS, o que evidencia a fragilidade do processo de construção social da saúde, que tem evoluído de maneira pontual (Gil, 2006).

Para tanto, é necessário que as equipes de saúde da família (EqSFs) cumpram um papel indutor na reorganização do processo de trabalho, cujo intuito é o de lidar com os desafios para a produção do cuidado: relações hierárquicas, práticas fragmentadas, pontuais, de baixa resolubilidade e centradas no atendimento individual (Gil, 2006; Santos et al., 2015). A organização do processo de trabalho na ESF é essencial para que a equipe possa contribuir na garantia da universalidade do acesso e na integralidade da atenção. Assim, além da competência técnica, os profissionais precisam ampliar o seu núcleo de saberes para as dimensões políticas e de gestão do trabalho em saúde, adotando habilidades de autogestão (Galavote et al., 2016). Isso lhes permite 
planejar e sistematizar o rol de ações de cuidados prestados à comunidade, bem como identificar problemas e definir prioridades no cotidiano laboral, em sintonia com a dinamicidade da assistência no âmbito da ESF (Cruz et al., 2014; Moreira et al., 2017a).

Desde o início da implantação da ESF até 2011, havia pouca informação sobre as realidades de organização do processo de trabalho, bem como do funcionamento e da estrutura das equipes (Rodrigues, Santos e Pereira, 2014). Desse modo, no citado ano, foi lançado o Programa Nacional de Melhoria do Acesso e da Qualidade da Atenção Básica (PMAQ-AB) (Brasil, 2013). Trata-se de uma avaliação dirigida à infraestrutura das unidades de saúde e às atividades e ações das EqSFs, que auxilia na identificação de áreas prioritárias e subsidia os gestores locais em melhorias na atenção à saúde da população. Embora o PMAQ-AB tenha limitações a discutir e enfrentar, esse programa traz em seu bojo um conjunto de esforços para a implementação de processos mais participativos de avaliação, assim como para a reengenharia nos processos de trabalho a partir do uso de informações (Teixeira et al., 2014; Silva et al., 2017; Chaves et al., 2018).

Segundo o Ministério da Saúde, "o PMAQ-AB situa a avaliação como estratégia permanente para tomada de decisão e ação central para melhoria da qualidade das ações de saúde, sendo esta considerada como atributo fundamental a ser alcançado no SUS" (Brasil, 2013, p. 10).

Por sua vez, a autoavaliação é assim definida:

A autoavaliação é entendida como ponto de partida da fase de desenvolvimento do PMAQ-AB, uma vez que os processos orientados para a melhoria da qualidade têm início na identificação e reconhecimento das dimensões positivas e também problemáticas do trabalho da gestão e das equipes de atenção à saúde. Nesse momento, deverão ser produzidos sentidos e significados com potencial de facilitar a mobilização de iniciativas para mudança e aprimoramento dos serviços (Brasil, 2013, p. 10).

Nesse enquadramento, tendo a organização do processo de trabalho como uma de suas subdimensões, o PMAQ-AB pode contribuir para a estruturação dos serviços e a reorientação do modelo de atenção à saúde, reconhecendo a AB como ordenadora do sistema (Rodrigues, Santos e Pereira, 2014; Medrado, Casanova e Oliveira, 2015; Silva et al., 2017). Para que esse potencial se consubstancie, são necessários investimentos e avaliações regulares, além de processos dialógicos entre os profissionais das equipes e das EqSFs com os gestores. Logo, os atores envolvidos devem se orientar por uma visão estratégica das dificuldades e conflitos existentes no contexto dos cuidados primários de saúde (Cruz et al., 2014; Medrado, Casanova e Oliveira, 2015; Silva et al., 2017). 
Nesse cenário, a autoavaliação das EqSFs constitui uma das iniciativas centrais da avaliação da $\mathrm{AB}$ e no debate acerca da mudança do modelo de atenção, tornando-se uma ferramenta benéfica para o aprimoramento da ESF (Teixeira et al., 2014). Essa autoavaliação pode ancorar o modo de reorganização do processo de trabalho e a gestão em saúde. Por meio da análise de fatores específicos das equipes, é possível planejar intervenções e elaborar estratégias concretas que superem os problemas constatados e garantam melhores avaliações da $\mathrm{AB}$ na ótica dos profissionais de saúde (Moreira, Vieira e Costa, 2016; Galavote et al., 2016).

Ademais, por meio do PMAQ-AB, as informações oriundas de momentos autoavaliativos das referidas equipes, que atuam em diferentes cenários, podem contribuir em avanços locais para a efetividade do cuidado, ao evidenciarem potencialidades, fragilidades e desafios no serviço (Souza et al., 2017). Na autoavaliação, a qualidade da AB pode ser mensurada pelo consenso dos profissionais da equipe, potencialmente habilitados para avaliar a realidade do processo de trabalho no contexto em que estão inseridos. Desse modo, torna-se pertinente analisar o processo de trabalho a partir da autoavaliação dos profissionais de saúde da família, no intuito de subsidiá-los na operacionalização e atuação de sua equipe. Tal análise também pode balizar a tomada de decisão e organização das práticas de cuidado, contribuindo para a consolidação da ESF.

Este estudo teve como objetivo avaliar a organização do processo de trabalho entre equipes de saúde da família de um município de Minas Gerais (MG), Brasil.

\section{Desenho metodológico}

A presente pesquisa segue o delineamento quantitativo, possui desenho transversal e analítico. Teve como cenário uma cidade polo, de grande porte, situada na Região Norte do Estado de Minas Gerais (MG), Brasil. Tal cidade tem contingente populacional estimado em 402.027 habitantes, caracterizase como um polo regional e é referência em áreas como prestação de serviços, comércio, educação e saúde. Os serviços da ESF compõem a rede de AB local desde a década de 1990 e, a partir de então, o número de EqSFs e a cobertura populacional por essas equipes têm crescido progressivamente, tornando o modelo de saúde da família a principal forma de organização da $\mathrm{AB}$ local (INSTITUTO BRASILEIRO DE GEOGRAFIA E ESTATÍSTICA, 2018).

Este estudo trata dos resultados da autoavaliação dos profissionais de saúde, durante o segundo ciclo do PMAQ-AB. No atual trabalho, foram incluídos os dados de 73 EqSFs, cadastradas no município, que foram coletados a partir de formulários da Autoavaliação para Melhoria do Acesso e da Qualidade da Atenção Básica (AMAQ), em site do Ministério da Saúde, impressos 
e disponibilizados por técnicos da Secretaria Municipal de Saúde para fins de pesquisa, em 2014.

A AMAQ é um instrumento referente à segunda fase de desenvolvimento do PMAQ-AB, desenvolvido pelo Ministério da Saúde brasileiro. É uma ferramenta de avaliação de serviços de saúde, gerada a partir de instrumentos validados, nacionalmente e internacionalmente. O instrumento é composto por dimensões e subdimensões relacionadas à equipe de $\mathrm{AB}$, com análise independente (Brasil, 2013).

Este trabalho abordou a subdimensão 'Organização do Processo de Trabalho', integrante da dimensão 'Educação Permanente, Processo de Trabalho e Atenção Integral à Saúde'. Essa subdimensão considera 14 padrões de qualidade, descritos no Quadro 1.

\section{Quadro 1}

Descrição dos 14 itens avaliados da subdimensão Organização do Processo de Trabalho, da Autoavaliação para Melhoria do Acesso e da Qualidade da Atenção Básica. Montes Claros, MG, Brasil, 2014

\begin{tabular}{l} 
A equipe de saúde da família: \\
\hline Trabalha com território definido, mantém vínculo com a população e se responsabiliza pela resolução das necessidades de \\
saúde. \\
Planeja suas ações com base no diagnóstico situacional de seu território e envolve a comunidade, no planejamento das \\
ações. \\
Organiza as agendas de atendimento individual dos diversos profissionais de forma compartilhada buscando assegurar a \\
ampliação do acesso e da atenção à saúde em tempo oportuno aos usuários. \\
Utiliza ferramentas para auxiliar na gestão do cuidado de casos complexos. \\
Realiza coordenação do cuidado dos usuários do seu território. \\
Faz registro e monitoramento das suas solicitações de exames, encaminhamentos às especialidades, bem como os retornos. \\
Utiliza estratégias ou ferramentas de abordagem familiar e de grupos familiares específicos. \\
Oferece atendimento à população de, no mínimo, 40 horas semanais. \\
Realiza visita domiciliar de maneira sistemática, programática, permanente e oportuna. \\
Realiza acolhimento à demanda espontânea. \\
Realiza o primeiro atendimento às urgências. \\
Realiza reuniões periódicas. \\
Realiza a alimentação do sistema de informação vigente da atenção básica de forma regular e consistente. \\
Realiza monitoramento e avaliação das ações e resultados alcançados.
\end{tabular}

Fonte: instrumento da Autoavaliação para Melhoria do Acesso e da Qualidade da Atenção Básica (Brasil, 2013)

Cada item permite avaliar o grau de adequação da organização do processo de trabalho nas unidades de saúde para as ações na $A B$, ou seja, avaliar o padrão de qualidade. Cada item é mensurado em escala de zero a 10 pontos, sendo zero o não cumprimento ao padrão (total inadequação da equipe) e 10 a total adequação. Os pontos são somados para classificar a equipe em padrão de qualidade: muito insatisfatório (0-15 pontos), insatisfatório (1631), regular (32-47), satisfatório (48-63) e muito satisfatório (64-80). Quanto maior a pontuação, melhor a classificação da equipe. A pontuação máxima na subdimensão estudada é de 80 pontos, ou seja, dez pontos para cada item (Brasil, 2013). Ainda, neste trabalho, as EqSFs foram categorizadas segundo localização urbana ou rural. Das 75 EqSFs, perderam-se informações referentes a duas equipes da zona urbana, portanto este trabalho se referiu aos resultados de 73 EqSFs.

Os dados foram organizados e analisados no Software IBM SPSS 22.0. $\mathrm{Na}$ análise estatística, primeiramente procedeu-se à descrição dos resulta- 
dos, apresentada em valores percentuais, média, desvio-padrão (DP), valores mínimo e máximo, percentis 25, 50 e 75. Processaram-se análises bivariadas para comparar as proporções de equipes em cada padrão de qualidade (muito insatisfatório a muito satisfatório) conforme localização (urbana, rural), pelo teste Likelihood ratio, alternativo do Teste Quiquadrado de Pearson. As médias de profissionais nas equipes foram comparadas conforme padrões de qualidade das equipes pelo teste Anova. A correlação entre os pontos obtidos pela equipe e o número de profissionais nas EqSFs foi efetuada pela correlação de Spearman. Os testes consideraram o nível de significância estatística $\mathrm{p}<0,05$.

Quanto aos aspectos éticos, o projeto de pesquisa que deu origem ao atual trabalho foi aprovado pelo Comitê de Ética em Pesquisa Envolvendo Seres Humanos da Universidade Estadual de Montes Claros (Unimontes), mediante o Parecer Consubstanciado n. 704.718/2014. Também se obteve a concordância institucional da Secretaria Municipal de Saúde.

\section{Avaliação da Organização do Processo de Trabalho na Saúde da Família: Resultados e Discussão}

Os achados do presente estudo permitiram evidenciar a autoavaliação das EqSFs quanto à organização do processo de trabalho no contexto da unidade de saúde da família. Optou-se por apresentar os resultados e suas respectivas discussões de forma conjunta, organizando-os nas seguintes seções: Organização do processo de trabalho na saúde da família: análise da autoavaliação das equipes; Classificação das equipes; Organização do processo de trabalho: equipes urbanas versus equipes rurais; Organização do processo de trabalho e número de profissionais nas equipes; Autoavaliação: implicações e perspectivas para a gestão da Atenção Básica.

\section{Organização do processo de trabalho na saúde da família: análise da auto-aliação das equipes}

A Tabela 1 apresenta a descrição dos padrões de qualidade da subdimensão avaliada, os quais, em sua maioria, foram bem atendidos. Entretanto, determinados quesitos auferiram resultados adversos. No item 'equipe trabalha com território definido, mantém vínculo com a população e se responsabiliza pela resolução das necessidades de saúde', houve concentração de 35,6\% das EqSFs nas pontuações sete e oito. Já no item 'coordenação do cuidado dos usuários do seu território', houve maior concentração nas pontuações seis e oito: $34,3 \%$ das equipes. Porém, em tais itens foram registradas expressivas frequências de equipes em total inadequação (com pontuação zero): 17,8\% e $13,7 \%$, respectivamente. Por sua vez, quanto ao 'registro e monitoramento das solicitações de exames, encaminhamentos às especialidades e retornos', a 
maior parte das equipes também obteve desempenho insatisfatório, concentrando-se na pontuação cinco $(23,3 \%)$.

Tabela 1

Distribuição das equipes de saúde da família conforme pontuação nos 14 padrões de qualidade da subdimensão Organização do Processo de Trabalho. Montes Claros, MG, Brasil, 2014

\begin{tabular}{|c|c|c|c|c|c|c|c|}
\hline Pontos & $\begin{array}{l}\text { A equipe... } \\
\text { Trabalha com } \\
\text { território } \\
\text { definido, } \\
\text { mantém } \\
\text { vínculo com a } \\
\text { população e } \\
\text { responsabiliza- } \\
\text { se pela } \\
\text { resolução das } \\
\text { necessidades } \\
\text { de saúde. }\end{array}$ & $\begin{array}{l}\text { Planeja ações } \\
\text { com base no } \\
\text { diagnóstico } \\
\text { situacional } \\
\text { do território } \\
\text { e envolve a } \\
\text { comunidade } \\
\text { no } \\
\text { planejamento } \\
\text { das ações. }\end{array}$ & $\begin{array}{l}\text { Organiza } \\
\text { agendas de } \\
\text { atendimento } \\
\text { individual } \\
\text { dos } \\
\text { profissionais } \\
\text { e assegura } \\
\text { a ampliação } \\
\text { do acesso e } \\
\text { da atenção } \\
\text { à saúde aos } \\
\text { usuários. }\end{array}$ & $\begin{array}{l}\text { Utiliza } \\
\text { ferramentas } \\
\text { para auxiliar } \\
\text { na gestão } \\
\text { do cuidado } \\
\text { de casos } \\
\text { complexos. }\end{array}$ & $\begin{array}{l}\text { Coordena } \\
\text { o cuidado } \\
\text { dos } \\
\text { usuários } \\
\text { do seu } \\
\text { território. }\end{array}$ & $\begin{array}{l}\text { Registra e } \\
\text { monitora as } \\
\text { solicitações } \\
\text { de exames, } \\
\text { encami- } \\
\text { nhamentos } \\
\text { às especia- } \\
\text { lidades e } \\
\text { retornos. }\end{array}$ & $\begin{array}{l}\text { Utiliza } \\
\text { estraté- } \\
\text { gias ou } \\
\text { ferramen- } \\
\text { tas de } \\
\text { abor- } \\
\text { dagem } \\
\text { familiar e } \\
\text { de grupos } \\
\text { familiares } \\
\text { específi- } \\
\text { cos. }\end{array}$ \\
\hline & $\mathrm{n}(\%)$ & $\mathrm{n}(\%)$ & $n(\%)$ & $\mathrm{n}(\%)$ & $\mathrm{n}(\%)$ & $\mathrm{n}(\%)$ & $\mathrm{n}(\%)$ \\
\hline 0 & $13(17,8)$ & $0(0,0)$ & $0(0,0)$ & $1(1,4)$ & $10(13,7)$ & $1(1,4)$ & $1(1,4)$ \\
\hline 1 & $1(1,4)$ & $0(0,0)$ & $0(0,0)$ & $0(0,0)$ & $2(2,7)$ & $1(1,4)$ & $0(0,0)$ \\
\hline 2 & $1(1,4)$ & $0(0,0)$ & $0(0,0)$ & $1(1,4)$ & $3(4,1)$ & $5(6,8)$ & $2(2,7)$ \\
\hline 3 & $8(11,0)$ & $0(0,0)$ & $3(4,1)$ & $1(1,4)$ & $5(6,8)$ & $6(8,2)$ & $2(2,7)$ \\
\hline 4 & $5(6,8)$ & $1(1,4)$ & $3(4,1)$ & $2(2,7)$ & $2(2,7)$ & $4(5,5)$ & $1(1,4)$ \\
\hline 5 & $7(9,6)$ & $0(0,0)$ & $12(16,4)$ & $4(5,5)$ & $9(12,3)$ & $17(23,3)$ & $5(6,8)$ \\
\hline 6 & $3(4,1)$ & $1(1,4)$ & $8(11,0)$ & $3(4,1)$ & $11(15,1)$ & $6(8,2)$ & $4(5,5)$ \\
\hline 7 & $14(19,2)$ & $2(2,7)$ & $16(21,9)$ & $10(13,7)$ & $9(12,3)$ & $14(19,2)$ & $13(17,8)$ \\
\hline 8 & $12(16,4)$ & $6(8,2)$ & $15(20,5)$ & $13(17,8)$ & $14(19,2)$ & $9(12,3)$ & $13(17,8)$ \\
\hline 9 & $5(6,8)$ & $17(23,3)$ & $13(17,8)$ & $17(23,3)$ & $6(8,2)$ & $6(8,2)$ & $16(21,9)$ \\
\hline 10 & $4(5,5)$ & $46(63,0)$ & $3(4,1)$ & $21(28,8)$ & $2(2,7)$ & $4(5,5)$ & $16(21,9)$ \\
\hline Pontos & $\begin{array}{l}\text { Oferece } \\
\text { atendimento à } \\
\text { população de, } \\
\text { no mínimo, } 40 \\
\text { horas sema- } \\
\text { nais. }\end{array}$ & $\begin{array}{l}\text { Realiza visita } \\
\text { domiciliar } \\
\text { de maneira } \\
\text { sistemática, } \\
\text { programática, } \\
\text { permanente e } \\
\text { oportuna. }\end{array}$ & $\begin{array}{l}\text { Realiza } \\
\text { acolhimento } \\
\text { à demanda } \\
\text { espontânea. }\end{array}$ & $\begin{array}{l}\text { Realiza o } \\
\text { primeiro } \\
\text { atendi- } \\
\text { mento às } \\
\text { urgências. }\end{array}$ & $\begin{array}{l}\text { Realiza } \\
\text { reuniões } \\
\text { periódi- } \\
\text { cas. }\end{array}$ & $\begin{array}{l}\text { Alimenta o } \\
\text { sistema de } \\
\text { informação } \\
\text { vigente da } \\
\text { atenção } \\
\text { básica } \\
\text { de forma } \\
\text { regular e } \\
\text { consistente. }\end{array}$ & $\begin{array}{l}\text { Monitora } \\
\text { e avalia } \\
\text { as ações e } \\
\text { resultados } \\
\text { alcança- } \\
\text { dos. }\end{array}$ \\
\hline & $\mathrm{n}(\%)$ & $\mathrm{n}(\%)$ & $\mathrm{n}(\%)$ & $\mathrm{n}(\%)$ & $\mathrm{n}(\%)$ & $n(\%)$ & $\mathrm{n}(\%)$ \\
\hline 0 & $4(5,5)$ & $1(1,4)$ & $0(0,0)$ & $1(1,4)$ & $0(0,0)$ & $0(0,0)$ & $0(0,0)$ \\
\hline 1 & $2(2,7)$ & $0(0,0)$ & $0(0,0)$ & $2(2,7)$ & $0(0,0)$ & $0(0,0)$ & $0(0,0)$ \\
\hline 2 & $1(1,4)$ & $0(0,0)$ & $1(1,4)$ & $2(2,7)$ & $1(1,4)$ & $1(1,4)$ & $0(0,0)$ \\
\hline 3 & $10(13,7)$ & $0(0,0)$ & $0(0,0)$ & $2(2,7)$ & $4(5,5)$ & $0(0,0)$ & $0(0,0)$ \\
\hline 4 & $9(12,3)$ & $1(1,4)$ & $1(1,4)$ & $2(2,7)$ & $1(1,4)$ & $3(4,1)$ & $1(1,4)$ \\
\hline 5 & $10(13,7)$ & $1(1,4)$ & $3(4,1)$ & $1(1,4)$ & $5(6,8)$ & $7(9,6)$ & $10(13,7)$ \\
\hline 6 & $9(12,3)$ & $3(4,1)$ & $3(4,1)$ & $5(6,8)$ & $5(6,8)$ & $8(11,0)$ & $9(12,3)$ \\
\hline 7 & $9(12,3)$ & $3(4,1)$ & $5(6,8)$ & $5(6,8)$ & $5(6,8)$ & $12(16,4)$ & $8(11,0)$ \\
\hline 8 & $7(11,0)$ & $19(26,0)$ & $17(23,3)$ & $14(19,2)$ & $15(20,5)$ & $13(17,8)$ & $17(23,3)$ \\
\hline 9 & $4(5,5)$ & $19(26,0)$ & $21(28,8)$ & $20(27,4)$ & $21(28,8)$ & $14(19,2)$ & $17(23,3)$ \\
\hline 10 & $7(9,6)$ & $26(35,6)$ & $22(30,1)$ & $21(28,8)$ & $16(21,9)$ & $15(20,5)$ & $11(15,1)$ \\
\hline
\end{tabular}

Fonte: Os autores 
Na Tabela 2, observa-se que a maior média de pontos foi para o 'planejamento de ações com base no diagnóstico situacional do território de atuação da equipe de saúde da família, contando com o envolvimento da comunidade adscrita' (9,38 pontos). Em contrapartida, o item relativo a 'trabalhar com território definido, manter vínculo com a população e responsabilizar-se pela resolução das necessidades de saúde' apresentou a menor média (5,19 pontos).

Tabela 2

Valores médios e separatrizes da pontuação das equipes de saúde da família nos 14 padrões de qualidade da subdimensão Organização do Processo de Trabalho. Montes Claros, MG, Brasil, 2014

\begin{tabular}{|c|c|c|c|c|c|c|c|}
\hline $\begin{array}{l}\text { Queståo } \\
\text { (A equipe...) }\end{array}$ & Média & Desvio-padrão & Mínimo & Máximo & $\begin{array}{l}\text { Percentis } \\
25\end{array}$ & $\begin{array}{l}\text { Percentis } \\
50\end{array}$ & $\begin{array}{l}\text { Percentis } \\
75\end{array}$ \\
\hline $\begin{array}{l}\text { Trabalha com } \\
\text { território definido, } \\
\text { mantém vínculo } \\
\text { com a população } \\
\text { e responsabiliza-se } \\
\text { pela resolução das } \\
\text { necessidades de } \\
\text { saúde. }\end{array}$ & 5,19 & 3,187 & 0 & 10 & 3,00 & 6,00 & 8,00 \\
\hline $\begin{array}{l}\text { Planeja suas } \\
\text { ações com base } \\
\text { no diagnóstico } \\
\text { situacional de seu } \\
\text { território e envolve } \\
\text { a comunidade no } \\
\text { planejamento das } \\
\text { ações. }\end{array}$ & 9,38 & 1,075 & 4 & 10 & 9,00 & 10,00 & 10,00 \\
\hline $\begin{array}{l}\text { Organiza as agendas } \\
\text { de atendimento } \\
\text { individual dos } \\
\text { diversos profissionais } \\
\text { de forma } \\
\text { compartilhada, } \\
\text { buscando assegurar a } \\
\text { ampliação do acesso } \\
\text { e da atenção à saúde } \\
\text { em tempo oportuno } \\
\text { aos usuários. }\end{array}$ & 6,96 & 1,767 & 3 & 10 & 5,50 & 7,00 & 8,00 \\
\hline $\begin{array}{l}\text { Utiliza ferramentas } \\
\text { para auxiliar na } \\
\text { gestão do cuidado de } \\
\text { casos complexos. }\end{array}$ & 8,05 & 2,094 & 0 & 10 & 7,00 & 9,00 & 10,00 \\
\hline $\begin{array}{l}\text { Realiza coordenação } \\
\text { do cuidado dos } \\
\text { usuários do seu } \\
\text { território. }\end{array}$ & 5,36 & 2,983 & 0 & 10 & 3,00 & 6,00 & 8,00 \\
\hline $\begin{array}{l}\text { Faz registro e } \\
\text { monitoramento das } \\
\text { suas solicitaçöes } \\
\text { de exames, } \\
\text { encaminhamentos às } \\
\text { especialidades, bem } \\
\text { como os retornos. }\end{array}$ & 5,89 & 2,343 & 0 & 10 & 5,00 & 6,00 & 8,00 \\
\hline
\end{tabular}




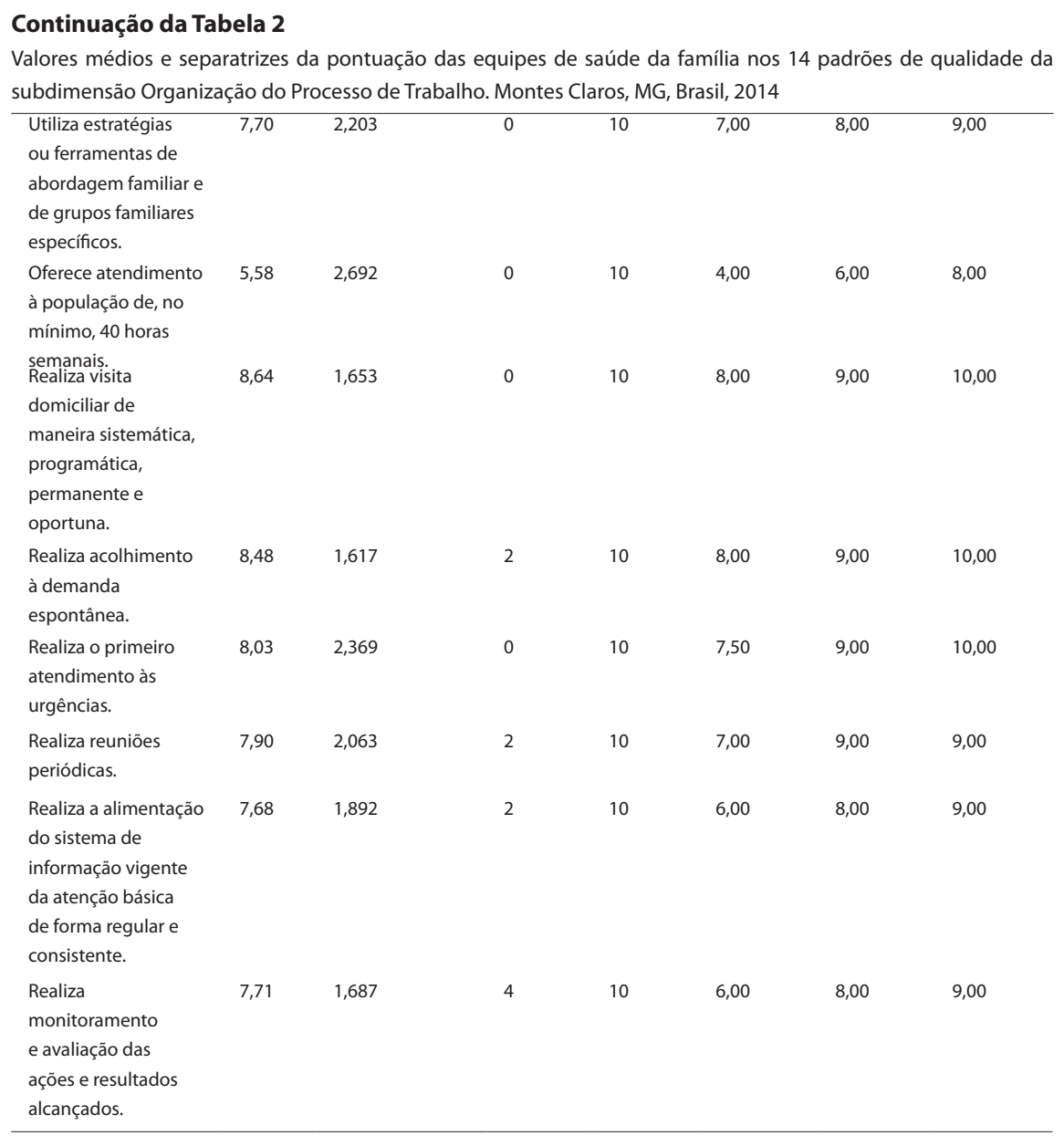

Fonte: Os autores

Nesta investigação, quanto ao item 'trabalhar com território definido, manter vínculo com a população e se responsabilizar pela resolução das necessidades de saúde', observaram-se resultados insatisfatórios entre as EqSFs. No caso da ESF, a territorialização persiste como um dos desafios a serem enfrentados. O território não é apenas a área de abrangência do serviço de saúde, mas agrega os espaços de poder, determinantes sociais do processo saúde-doença-cuidado e equipamentos sociais. Tal configuração deve integrar o rol de conhecimentos da equipe (Garcia et al., 2014) para que ela possa acompanhar os usuários, ao longo do tempo, por meio da vigilância constante das diversas condições de saúde (Souza et al., 2017).

Por sua vez, o vínculo constitui um elemento essencial para a adesão dos usuários aos serviços e ações de saúde, pois permite que se sintam mais seguros, ouvidos e cuidados. Então, torna-se possível compor a corresponsabilização com a assistência e a produção do cuidado comprometi- 
do e que vai além da dimensão biológica da doença (Santos et al., 2015). Portanto, os componentes integrantes do citado item são relevantes, posto que se relacionam com o planejamento da demanda programada e com o monitoramento territorializado da população adscrita. Desse modo, as EqSFs podem se fundamentar nas informações do PMA-AB para prestar uma atenção que supere práticas fragmentadas, voltadas ao cumprimento de tarefas mecânicas e à quantificação dos atendimentos (Garnelo et al., 2014).

A coordenação do cuidado dos usuários do território cadastrados na ESF, bem como o registro e monitoramento das solicitações de exames e encaminhamentos e retornos às especialidades, foi outro item com situação inadequada neste estudo. Desse modo, conforme Bousquat et al. (2017a), tais achados sinalizam a fragilidade da $\mathrm{AB}$ em se instituir como porta de entrada prioritária na rede do sistema público de saúde, bem como sua dificuldade em proporcionar a resolutividade no cuidado em saúde. A incipiente coordenação da assistência pela $\mathrm{AB}$ tem sido constatada em pesquisas nacionais (Protasio et al., 2014; Carneiro et al., 2014; Seidl et al., 2014; Fontana, Lacerda e Machado, 2016; Uchôa et al., 2016; Souza et al., 2017; Almeida, Marin e Casotti, 2017; Bousquat et al., 2017a; Chaves et al., 2018) e internacionais (Haggerty et al., 2003; Hudon et al., 2015; Lamothe, Sylvain e Sit, 2015; Tarrant et al., 2015).

Trata-se de um atributo central para o manejo de usuários na rede de atenção à saúde (Hudon et al., 2015; Lamothe, Sylvain e Sit, 2015; Bousquat et al., 2017a), que abarca a sincronicidade de ações de acolhimento, encaminhamento, monitoramento e responsabilização, independentemente do ponto da rede onde esteja o usuário (Seidl et al., 2014; Fontana, Lacerda e Machado, 2016). Para tal, requer-se uma AB robusta, capaz de coordenar o percurso terapêutico desse usuário e facilitar a prestação de serviços em local e tempo oportunos (Tarrant et al., 2015; Bousquat et al., 2017a), mantendo um processo com responsabilização e vínculo com o cliente (Uchôa et al., 2016). Ressalta-se que as fragilidades na integração da rede de atenção à saúde afetam a integralidade e o papel da ESF como coordenadora do cuidado e ordenadora das redes (Protasio et al., 2014).

Contudo, é válido ponderar que a consolidação da coordenação do cuidado tem sido um nó crítico em sistemas nacionais de saúde universais em diversos países, comprometendo a organicidade e a concretude necessárias ao pleno funcionamento desses sistemas. No Brasil, por sua vez, a proposição da conformação de redes de atenção à saúde ainda é relativamente recente. $\mathrm{Ob}$ teve relevância política no início do século XXI, almejando o enfrentamento da fragmentação da atenção, a ampliação do acesso, a busca da equidade e da universalidade. A integração entre os pontos dessas redes é uma diretriz de notável complexidade, ao se considerar aspectos mais amplos, referentes à 
regionalização da saúde no país; às desigualdades e às diversidades regionais e, até mesmo, no interior dos municípios; à amplitude das atribuições do Estado no setor saúde; ao arranjo federativo trino; e à heterogeneidade de atores - governamentais e não governamentais, públicos e privados - responsáveis pela prestação de serviços de saúde (Bousquat et al., 2017a).

Nesse contexto, é imperativo que o sistema de saúde reconheça o papel da ESF e a necessidade de efetiva articulação entre atenção primária, secundária e terciária. Os serviços, em cada nível de atenção, devem estar organizados para atender satisfatoriamente às necessidades dos usuários (Starfield, 2002; Carneiro et al., 2014). No intuito de que se consolidem a universalização do acesso e a qualidade na atenção à saúde (Haggerty et al., 2003; Bousquat et al., 2017a), a gestão deve dar condições para que as EqSFs possam coordenar as ações de saúde, atuando de maneira interligada com os profissionais dos serviços especializados da rede de atenção à saúde (Seidl et al., 2014; Fontana, Lacerda e Machado, 2016).

\section{Classificação das equipes}

A partir do somatório da pontuação, obtida pelas equipes a partir da autoavaliação dos profissionais, classificaram-se as EqSFs em quatro padrões de qualidade, como demonstrado na Tabela 3. A maior parte foi classificada no padrão satisfatório (56,2\%), seguido de muito satisfatório para 30,1\%, enquanto $12,3 \%$ das equipes se situaram no nível regular.

Tabela 3

Classificação das equipes de saúde da família na subdimensão Organização do Processo de Trabalho. Montes Claros, MG, Brasil, 2014

\begin{tabular}{lcc}
\hline \multicolumn{3}{c}{ Organização do processo de trabalho } \\
\hline Classificação & $\mathbf{n}$ & $\%$ \\
\hline Muito insatisfatório & 1 & 1,4 \\
Regular & 9 & 12,3 \\
Satisfatório & 41 & 56,2 \\
Muito satisfatório & 22 & 30,1 \\
Total & 73 & 100,00 \\
\hline Fonte: Os autores & &
\end{tabular}

Fonte: Os autores

Em linhas gerais, ainda que a maior parte das EqSFs integrantes desta investigação tenha se situado em uma boa classificação e atingido altas pontuações nos itens integrantes da subdimensão aqui abordada, quantidade importante foi classificada em níveis muito insatisfatórios e regulares. À luz da literatura sobre avaliação da $\mathrm{AB}$ por meio do $\mathrm{PMAQ}-\mathrm{AB}$, pode-se considerar que essa situação foi similar à encontrada em outros serviços de saúde da família. São eles: em um município do Estado de Minas Gerais (Moreira, Vieira e Costa, 2016), em municípios do Estado de Santa Catarina (Fontana, 
Lacerda e Machado, 2016), do Espírito Santo (Garcia et al., 2014), em regiões de saúde do Estado do Rio de Janeiro (Medrado, Casanova e Oliveira, 2015) e no Brasil (Cruz et al., 2014).

Considera-se necessário investir e apoiar as equipes que não auferiram avaliações positivas, para que possam ofertar uma assistência mais qualificada aos usuários (Moreira, Vieira e Costa, 2016). Também é pertinente recomendar que os profissionais reconheçam a importância que os itens avaliados exercem no cotidiano laboral, para que aprimorem, cada vez mais, o seu processo de trabalho (Carneiro et al., 2014).

Garcia et al. (2014) assinalam que o trabalho das EqSFs ainda é estruturado de maneira verticalizada, baseado em instrumentos pouco reflexivos. Torna-se necessário considerar a centralidade do saber-fazer dos profissionais que operam o cuidado e as demandas de saúde da comunidade assistida, na perspectiva de difusão das práticas de planejamento e na organização do dia a dia dessas equipes. Sendo assim, ressalta-se a premência de realizar essas práticas, periodicamente (Rodrigues, Santos e Pereira, 2014), uma vez que podem contribuir substancialmente para o alcance dos objetivos da ESF, como descentralização e capilaridade, universalidade, resolutividade, acessibilidade, vínculo, continuidade do cuidado e integralidade da atenção (Garnelo et al., 2014).

\section{Organização do processo de trabalho: equipes urbanas versus equipes rurais}

Ainda, as EqSFs foram categorizadas e comparadas em cada padrão de qualidade, conforme localização urbana ou rural. Foi identificada diferença estatisticamente significante entre as equipes da zona urbana e rural, com melhor situação para as de localização urbana $(p=0,001)$. E somente uma equipe rural obteve classificação muito satisfatória (Tabela 4).

Tabela 4

Classificação das equipes de saúde da família na subdimensão Organização do Processo de Trabalho conforme localização urbana ou rural. Montes Claros, MG, Brasil, 2014

\begin{tabular}{lllll}
\hline \multicolumn{5}{c}{ Localização da equipe } \\
\hline Classificação & $\begin{array}{l}\text { Zona urbana } \\
\mathrm{n}(\%)\end{array}$ & $\begin{array}{l}\text { Zona rural } \\
\mathrm{n}(\%)\end{array}$ & $\begin{array}{l}\text { Total } \\
\mathrm{n}(\%)\end{array}$ & $\begin{array}{l}\text { Likelihood Ratio } \\
\mathrm{p}\end{array}$ \\
\hline Muito Insatisfatório & $1(100,0)$ & $0(0,0)$ & $1(100,0)$ & \\
\hline Regular & $3(33,3)$ & $6(66,7)$ & $9(100,0)$ & 0,001 \\
\hline Satisfatório & $37(90,2)$ & $4(9,8)$ & $41(100,0)$ & $22(100,0)$ \\
\hline Muito satisfatório & $21(95,5)$ & $1(4,5)$ & $73(100,0)$ & \\
\hline Total & $62(84,9)$ & $11(15,1)$ & &
\end{tabular}


$\mathrm{Na}$ atual investigação, ao se mensurar os padrões de qualidade da $\mathrm{AB}$ segundo a localização rural e urbana, foram registrados melhores resultados entre as equipes situadas na zona urbana, similarmente ao observado em trabalhos prévios (Moreira, Vieira e Costa, 2016; Moreira et al., 2017a). É válido informar que, mesmo no interior dos municípios, podem existir unidades de saúde com distintas realidades populacionais e de organização dos seus serviços (Fontana, Lacerda e Machado, 2016). Supõe-se que, na área rural, as barreiras geográficas, aqui demarcadas pela maior distância geográfica entre a residência do usuário e a unidade de saúde da ESF, compõem um elemento que afeta o acesso aos serviços de saúde e fragiliza a assistência integral (Moreira, Vieira e Costa, 2016).

Fausto et al. (2014) advertem que as diferenças verificadas nas condições de oferta e uso de serviços da ESF, principalmente em áreas rurais e remotas, sinalizam novos desafios e demandas para os profissionais de saúde da família e para a gestão municipal, os quais ficam incumbidos por atender a todo o conjunto de tarefas da $\mathrm{AB}$. Assim, na formulação de políticas e programação em saúde, é preciso adotar mecanismos de enfrentamento do problema da qualidade e resolutividade das unidades básicas de saúde, em todos os cenários, para ampliar os serviços disponibilizados e promover o acesso oportuno - porta aberta e resolutiva. Por conseguinte, todas as EqSFs, em qualquer localização geográfica, terão os subsídios necessários para desenvolver e organizar seu processo de trabalho e, desse modo, garantir a atenção integral ao usuário do SUS.

\section{Organização do processo de trabalho e número de profissionais nas equipes}

A análise de correlação entre o número de profissionais e a pontuação das EqSFs na subdimensão Organização do Processo de Trabalho demonstrou que as categorias profissionais cirurgião-dentista $(p=0,001)$ e auxiliar e técnico em odontologia ( $\mathrm{p}=0,003)$ se correlacionaram positivamente e melhor explicaram a variação de pontos entre as equipes (Tabela 5). Todas as equipes de saúde apresentaram, igualmente, um profissional de enfermagem; logo, não houve variabilidade dos dados entre elas.

Tabela 5

Correlação entre o número de profissionais nas equipes de saúde da família e pontos na subdimensão Organização do Processo de Trabalho. Montes Claros, MG, Brasil, 2014

\begin{tabular}{llll}
\hline Número de profissionais por equipe & \multicolumn{2}{l}{ Pontos na subdimensão Organização do Processo de Trabalho } \\
\hline Categoria (n' ${ }^{\circ}$ mínimo-máximo) & $\mathbf{R}$ & $\mathbf{R}^{\mathbf{2}}$ & $\mathbf{p}$ \\
\hline Profissionais no geral (8-11) & 0,084 & 0,007 & 0,681 \\
Técnicos em enfermagem (0-1) & 0,100 & 0,01 & 0,661 \\
Médicos (0-1) & 0,188 & 0,035 & 0,059 \\
Cirurgiões-dentistas (0-1) & 0,209 & 0,043 & 0,001 \\
Auxiliarese técnicos em odontologia (0-2) & 0,229 & 0,052 & 0,003 \\
Agentes comunitários de saúde (4-11) & $-0,151$ & 0,022 & 0,127 \\
\hline
\end{tabular}

Fonte: Os autores 
Neste trabalho, outro resultado interessante constatado foi que o maior número de profissionais cirurgiões-dentistas e auxiliares e técnicos de odontologia nas equipes investigadas apresentou uma correlação linear positiva e estatisticamente significante com a maior pontuação na subdimensão. Logo, tal achado sugere melhor qualidade da $\mathrm{AB}$ na autoavaliação dos membros da equipe de saúde da família, segundo Moreira, Vieira e Costa (2016). Outras pesquisas também encontraram associação entre presença desses profissionais na equipe e melhor avaliação da AB (Uchôa et al., 2016; Moreira, Vieira e Costa, 2016; Moreira et al., 2017a).

Tal achado sinaliza que a equipe multiprofissional completa obtém melhores resultados no processo de trabalho (Moreira; Vieira; Costa, 2016), além de propiciar ações mais compartilhadas entre os membros da equipe e maior elenco de cuidados. Costa et al. (2014) elucidam que, historicamente, a categoria profissional da área da odontologia se estabeleceu em ambiente clínico em quatro paredes, prestando uma assistência individual, com orientação biologicista e tecnicista. No entanto, face à associação aqui evidenciada, pode-se aludir, no cenário da ESF, a uma superação do modelo tradicional de atenção à saúde e a efetivação de um novo paradigma nessa profissão, orientado pelo cuidado à saúde da família e pela promoção da saúde.

Em adição, Bousquat et al. (2017b) assinalam que equipe de saúde bucal assume notável valor no cenário da ESF, dada a sua importância nas políticas de saúde brasileiras nos últimos anos, com a execução de diversos programas específicos, o que contribui para assegurar a integralidade da atenção. Convém ressaltar que a estratégia brasileira de ofertar o acesso ao cuidado em saúde bucal no mesmo espaço das demais práticas de saúde na $A B$, em unidade de saúde da família, tem sido vista internacionalmente como bastante positiva; entretanto, a falta de profissionais dessa categoria diminui o rol de potencialidades das EqSFs. Além disso, pode gerar atraso na prestação da assistência bucal, levando a sérios agravos e doenças, com impacto na qualidade de vida e na saúde dos usuários (Jatrana, Crampton e Filoche, 2009; Emami et al., 2016; Bousquat et al., 2017b).

\section{Autoavaliação: implicações e perspectivas para a gestão da Atenção Básica}

Face à realidade delineada na presente avaliação da $\mathrm{AB}$, espera-se que este estudo possa subsidiar empreendimentos de autoavaliação que propiciem às EqSFs e aos gestores a autorreflexão sobre o processo de trabalho, com vistas à identificação dos problemas e à formulação de estratégias para melhoria das práticas e das relações nos serviços da ESF. A AB abrange processos complexos e dinâmicos, com diversidade de atribuições e alta pressão por resultados bem-sucedidos. Daí emerge a necessidade de vincular o planejamento com 
a avaliação de forma mais participativa e negociada com as equipes (Cruz et al., 2014), a fim de que o PMAQ-AB, inserido no escopo de políticas do Ministério da Saúde, possa efetivamente induzir melhorias na AB (Fontana, Lacerda e Machado, 2016).

Seguindo a perspectiva de Moreira et al. (2017a) e Moreira, Vieira e Costa (2016), os resultados da autoavaliação das EqSFs aqui abordados demandam um processo contínuo de educação permanente e de autoavaliação, articulado à realidade das equipes, bem como às reais demandas e características dos serviços e de suas respectivas comunidades adscritas. Logo, torna-se premente planejar ações de apoio a essas equipes e de incentivo à qualificação profissional, permanentemente e no contexto do serviço, no intuito de promover o desenvolvimento de competências e de habilidades específicas do cuidado na AB.

Também recomenda-se aos gestores o reconhecimento da avaliação como subsídio indispensável ao planejamento de ações, o qual permite uma visão da operacionalidade da ESF, dos aspectos facilitadores e dos nós críticos que prejudicam a boa qualidade da atenção. Assim, torna-se possível um planejamento que possa retificar as inadequações observadas na autoavaliação das EqSFs. Isso torna-se indubitavelmente necessário, uma vez que tais inadequações restringem o potencial dos serviços para a consolidação da ESF, na concepção da reorganização de práticas de cuidado direcionadas à tríade indivíduo-família-comunidade e à qualidade da atenção à saúde (Moreira et al., 2017b).

\section{Limitações do estudo}

Por fim, é preciso informar que os resultados deste trabalho devem ser considerados à luz de certas limitações. Tratou-se de uma pesquisa transversal, sendo que esse delineamento impede definir uma relação de causalidade para as associações registradas. Os achados foram circunscritos ao cenário das unidades da ESF de um município. Ainda, não foram aplicadas técnicas de observação direta, mas, sim, investigados dados procedentes da autoavaliação das EqSFs, os quais podem estar sujeitos a um possível viés de informação.

\section{Conclusão}

O presente estudo evidenciou a autoavaliação das EqSFs quanto à organização do processo de trabalho. No geral, foi observada uma boa análise por parte dessas equipes; porém, resultados insatisfatórios foram observados em importantes componentes do cuidado na $\mathrm{AB}$, no que concerne a atuar com território definido, vínculo e responsabilização pelo atendimento às necessidades de saúde da comunidade. A coordenação do cuidado, bem como o 
registro e monitoramento das solicitações de exames, encaminhamentos às especialidades e retornos também foram itens que ficaram aquém do desejável. Desse modo, atenção especial e apoio devem ser dados àquelas equipes que ficaram no patamar insatisfatório e com baixa pontuação nos citados itens.

Nas análises, averiguaram-se resultados interessantes: houve melhor classificação para as equipes da zona urbana e com profissionais cirurgiões-dentistas e auxiliares e técnicos em odontologia em sua composição. A integralidade e a qualidade do cuidado, bem como boas condições de trabalho para os profissionais, devem ser resguardadas para todas as EqSFs, independentemente se localizadas em áreas remotas ou não. Já o achado referente à categoria profissional da odontologia constitui aspecto positivo, posto que uma equipe completa tem maior preparo para uma melhor performance e atenção à saúde da comunidade.

Assim sendo, espera-se que os achados desta pesquisa possam agregar novas evidências sobre a avaliação dos serviços e das equipes de $\mathrm{AB}$. Por meio de novo conhecimento sobre o processo de autoavaliação pelos profissionais da ESF, podem-se sinalizar subsídios para o aprimoramento da organização do processo de trabalho e para a contínua qualificação da prática das equipes, com vistas à melhor organização da assistência e à qualidade do cuidado. Tais achados também podem ser pertinentes na perspectiva de ancorar os gestores do sistema de saúde local no fortalecimento da atenção ofertada no nível primário da rede de atenção à saúde.

\section{Colaboradores}

Cássio de Almeida Lima participou da concepção do estudo, do planejamento e da coleta de dados, da interpretação dos dados, da redação e revisão do artigo. Kênia Souto Moreira participou da concepção do estudo, do planejamento e da coleta de dados, da elaboração do banco de dados, da análise e da interpretação dos dados e da redação do artigo. Gustavo Silva Costa e Rayane Soares Maia participaram da concepção do estudo, da coleta de dados, da elaboração do banco de dados e contribuíram na redação do artigo. Mânia de Quadros Coelho Pinto participou da concepção do estudo, do planejamento e da coleta de dados, da análise dos dados e da redação do artigo. Maria Aparecida Vieira colaborou na orientação, participou da concepção do estudo, do planejamento da coleta de dados, da interpretação dos dados e da revisão crítica do artigo. Simone de Melo Costa orientou todas as etapas do estudo e da elaboração do artigo, participou da concepção do estudo, do planejamento da coleta de dados, da análise e interpretação dos dados, da 
redação e da revisão crítica do artigo. Todos os autores leram e aprovaram a versão final do artigo.

\section{Financiamento}

Conselho Nacional de Desenvolvimento Científico e Tecnológico (CNPq), Processo 437324/2016-8 (auxílio financeiro para Pós-doutorado realizado pela autora Simone de Melo Costa).

\section{EVALUACIÓN DEL PROCESO DE TRABAJO ENTRE EQUIPOS DE SALUD DE LA FAMILIA DE UN MUNICIPIO DE MINAS GERAIS, BRASIL}

Resumen Este estudio tuvo como objetivo evaluar la organización del proceso de trabajo entre equipos de salud de la familia de un municipio del Estado de Minas Gerais, Brasil. Investigación cuantitativa, transversal, analítica, desarrollada en el 2014. Se investigaron formularios de Autoevaluación para Mejora del Acceso y la Calidad de la Atención Básica de 73 equipos. Se consideraron 14 puntos de la subdimensión organización del proceso de trabajo, evaluados con valores entre cero y diez puntos, correspondiendo respectivamente a la total inadecuación o adecuación del equipo al estándar evaluado. Los equipos se clasificaron desde el estándar 'muy insatisfactorio' hasta el 'muy satisfactorio'. Se efectuaron análisis estadísticos con nivel de significación p<0,05. El 86,3\% se clasificó en los estándares muy satisfactorio/satisfactorio y el 13,7\% como regular/muy insatisfactorio. Tres puntos - 'Territorio definido, vínculo con la población y responsabilidad por la resolución de las necesidades de salud'; 'Coordinación del cuidado' y 'Monitoreo de las solicitudes de exámenes' - presentaron un rendimiento inadecuado. Los equipos de la zona urbana tuvieron un mejor resultado. El mayor número de profesionales de odontología estuvo correlacionado a la mayor puntuación. Se debe dar atención especial a aquellos equipos con baja puntuación. Se debe poner énfasis en una mejor organización del proceso de trabajo, a fin de optimizar el rendimiento en el cuidado de la salud de la comunidad.

Palavras clave gestión en salud; servicios de salud; evaluación de servicios de salud; estrategia de salud de la familia; atención primaria de la salud. 


\section{Referências}

ALMEIDA, Patty F.; MARIN, Juliana; CASOTTI, Elisete. Estratégias para consolidação da coordenação do cuidado pela Atenção Básica. Trabalho, Educação e Saúde, Rio de Janeiro, v. 15, n. 2, p. 373-398, 2017.

BOUSQUAT, Aylene et al. Primary health care and the coordination of care in health regions: managers' and users' perspective. Ciência \& Saúde Coletiva, Rio de Janeiro, v. 22, n. 4, p. 1.141-1.154, 2017a.

BOUSQUAT, Aylene et al. Tipologia da estrutura das unidades básicas de saúde brasileiras: os 5 R. Cadernos de Saúde Pública, Rio de Janeiro, v. 33, n. 8, p. 1-14, $2017 \mathrm{~b}$.

INSTITUTO BRASILEIRO DE GEOGRAFIA E ESTATÍSTICA (IBGE). Cidades@. Brasil. Minas Gerais. Montes Claros. Rio de Janeiro: IBGE, 2018. Disponível em: <https://cidades.ibge. gov.br/brasil/mg/montes-claros/panorama $>$. Acesso em: 18 jan. 2018.

BRASIL. Ministério da Saúde. Secretaria de Atenção à Saúde. Departamento de Atenção Básica. $A M A Q$ : autoavaliação para melhoria do acesso e da qualidade. Brasília: Ministério da Saúde, 2013.

CARNEIRO, Maria S. M. et al. Avaliação do atributo coordenação da Atenção Primária à Saúde: aplicação do PCATool a profissionais e usuários. Saúde em Debate, Rio de Janeiro, v. 38, n. esp., p. 279-295, 2014.

CHAVES, Lenir A. et al. Integração da atenção básica à rede assistencial: análise de componentes da avaliação externa do PMAQ-AB. Cadernos de Saúde Pública, Rio de Janeiro, v. 34, n. 2, p. 1-16, 2018.

COSTA, Simone de M. et al. Práticas de trabalho no âmbito coletivo: profissionais da equipe Saúde da Família. Cadernos de Saúde Coletiva, Rio de Janeiro, v. 22, n. 3, p. 292-299, 2014.

CRUZ, Marly M. da et al. Usos do planejamento e autoavaliação nos processos de trabalho das equipes de Saúde da Família na Atenção Básica. Saúde em Debate, Rio de Janeiro, v. 38, n. esp., p. 124-139, 2014.

EMAMI, Elham et al. Integration of oral health into primary care: a scoping review protocol. BMJ Open, London, v. 6, n. 10, p. 1-5, 2016.

FAUSTO, Márcia C. R. et al. A posição da Estratégia Saúde da Família na rede de atenção à saúde na perspectiva das equipes e usuários participantes do PMAQ-AB. Saúde em Debate, Rio de Janeiro, v. 38, n. esp., p. 13-33, 2014.

FONTANA, Karine C.; LACERDA, Josimari T. de; MACHADO, Patrícia M. de O. O processo de trabalho na Atenção Básica à saúde: avaliação da gestão. Saúde em Debate, Rio de Janeiro, v. 40, n. 110, p. 64-80, 2016.

GALAVOTE, Heletícia S. et al. The nurse's work in primary health care. Escola Anna Nery Revista de Enfermagem, Rio de Janeiro, v. 20, n. 1, p. 90-98, 2016.

GARCIA, Ana C. P. et al. Análise da organização da Atenção Básica no Espírito Santo: (des)velando cenários. Saúde em Debate, Rio de Janeiro, v. 38, n. esp., p. 221-236, 2014.

GARNELO, Luiza et al. Organização do cuidado às condições crônicas por equipes de Saúde da Família na Amazônia. Saúde em Debate, Rio de Janeiro, v. 38, n. esp., p. 158-172, 2014.

GIL, Célia R. R. Atenção primária, atenção básica e saúde da família: sinergias e singularidades do contexto brasileiro. Cadernos de Saúde Pública, Rio de Janeiro, v. 22, n. 6, p. 1171-1181, 2006.

HAGGERTY, Jeannie L. et al. Continuity of care: a multidisciplinary review. BMJ, London, v. 327, n. 7425, p. 1219-1221, 2003.

HUDON, Catherine et al. Case management in primary care for frequent users of health care services with chronic diseases: a qualitative 
study of patient and family experience. Annals of Family Medicine, Cleveland, v. 13, n. 6, p. 523-528, 2015.

JATRANA, Santosh; CRAMPTON, Peter; FILOCHE, Sara K. The case for integration oral health into primary care. The New Zealand Medical Journal, Wellingtonv, v. 122, n. 1301, p. 43-52, 2009.

LAMOTHE, Lise; SYLVAIN, Chantal; SIT, Vanessa. Multimorbidity and primary care: emergence of new forms of network organization. Santé Publique, Paris, v. 27, supl. 1, p. S129-135, 2015.

LEITE, Raquel S. et al. Estratégia Saúde da Família versus centro de saúde: modalidades de serviços na percepção do usuário. Cadernos Saúde Coletiva, Rio de Janeiro, v. 24, n. 3, p. 323-329, 2016.

LIMA, Cássio de A. et al. Relação profissionalusuário de saúde da família: perspectiva da bioética contratualista. Revista Bioética, Brasília, v. 22, n. 1, p. 152-160, 2014.

MACIEL, Antônio G.; CALDEIRA, Antônio P.; DINIZ, Francisco J. L. de S. Impacto da Estratégia Saúde da Família sobre o perfil de morbidade hospitalar em Minas Gerais. Saúde em Debate, Rio de Janeiro, v. 38, n. esp., p. 319-330, 2014.

MEDRADO, Josiane R. S.; CASANOVA, Angela O.; OLIVEIRA, Cátia C. M. de. Estudo avaliativo do processo de trabalho das Equipes de Atenção Básica a partir do PMAQ-AB. Saúde em Debate, Rio de Janeiro, v. 39, n. 107, p. 1.033-1.043, 2015.

MOREIRA, Kênia S. et al. Assessment of infrastructure of family health units and equipment used in primary care actions. Cogitare Enfermagem, Curitiba, v. 22, n. 2, p. e51283, 2017 b.

MOREIRA, Kênia S. et al. Educação permanente e qualificação profissional para Atenção Básica. Revista Saúde e Pesquisa, Maringá, v. 10, n. 1, p. 101-109, 2017a.
MOREIRA, Kênia S.; VIEIRA, Maria A.; COSTA, Simone de M. Qualidade da Atenção Básica: avaliação das equipes de saúde da família. Saúde em Debate, Rio de Janeiro, v. 40, n. 111, p. 117-127, 2016.

PROTASIO, Ane. P. L. et al. Avaliação do sistema de referência e contrarreferência do estado da Paraíba segundo os profissionais da Atenção Básica no contexto do $1^{\circ}$ ciclo de Avaliação Externa do PMAQ-AB. Saúde em Debate, Rio de Janeiro, v. 38, n. esp., p. 209-220, 2014

RODRIGUES, Valéria; SANTOS, Cleber R. I. dos; PEREIRA, Mariana U. A experiência de planejar e operacionalizar o PMAQ-AB no estado do Acre. Saúde em Debate, Rio de Janeiro, v. 38, n. esp., p. 173-181, 2014.

SANTOS, Adriano M. dos et al. Desafios à gestão do trabalho e educação permanente em saúde para a produção do cuidado na Estratégia Saúde da Família. Revista de APS, Juiz de Fora, v. 18, n. 1, p. 39-49, 2015.

SEIDL, Helena et al. Gestão do trabalho na Atenção Básica em Saúde: uma análise a partir da perspectiva das equipes participantes do PMAQ-AB. Saúde em Debate, Rio de Janeiro, v. 38, n. esp., p. 94-108, 2014.

SILVA, Lais M. C. et al. Implementation of the Improved Access and Quality Program according to Primary Care managers in São Paulo. Acta Paulista de Enfermagem, São Paulo, v. 30, n. 4, p. 397-403, 2017.

SOUZA, Miriam F. de et al. Care coordination in PMAQ-AB: an Item Response Theory-based analysis. Revista de Saúde Pública, São Paulo, v. 51, p. 1-10, 2017.

STARFIELD, Bárbara. Atenção Primária: equilíbrio entre necessidades de saúde, serviços e tecnologia. Brasília: UNESCO, Ministério da Saúde, 2002.

TARRANT, Carolyn et al. "Falling through gaps": primary care patients' accounts of breakdowns in experienced continuity of 
care. Family Practice, Oxford, v. 32, n. 1, p. 82-87, 2015.

TEIXEIRA, Mirna B. et al. Avaliação das práticas de promoção da saúde: um olhar das equipes participantes do Programa Nacional de Melhoria do Acesso e da Qualidade da Atenção
Básica. Saúde em Debate, Rio de Janeiro, v. 38, n. esp., p. 52-68, 2014.

UCHÔA, Severina A. da C. et al. Potential access to primary health care: what does the National Program for Access and Quality Improvement data show?. Revista LatinoAmericana de Enfermagem, Ribeirão Preto, v. 24, p. 1-28, 2016. 